\title{
Microlensing evidence for super-Eddington disk accretion in quasars
}

\author{
Nikolai I. Shakura and Pavel Abolmasov \\ Sternberg Astronomical Institute \\ Universitetsky pr., 13, Moscow 119991, Russia \\ email: pavel.abolmasov@gmail.com
}

\begin{abstract}
Gravitational microlensing by the stellar population of lensing galaxies provides an important opportunity to spatially resolve the accretion disk structure in strongly lensed quasars. Some of the objects (like Einstein's cross) are reasonably consistent with the predictions of the standard accretion disk model. In other cases, the size of the emitting region is larger than predicted by the standard thin disk theory and practically independent on wavelength. This may be interpreted as an observational manifestation of an optically-thick scattering envelope possibly related to super-Eddington accretion with outflows.
\end{abstract}

Keywords. accretion, accretion discs, gravitational lensing: micro, quasars: general

\section{Introduction}

The optical/UV angular sizes of quasar accretion disks at $z \gtrsim 1$ are $\sim 0.1 \mu$ as. These sizes may be resolved only by indirect methods such as microlensing by the stellar population of lensing galaxies (Wambsganss 2006). Fortunately, the Einstein-Chwolson radii of Solar-mass microlenses at $\sim$ Gpc distances are of the same order, and all the microlensing curves are sensitive to the angular size of the source.

Disk size estimates based on microlensing effects show several features that are difficult to interpret in the framework of standard thin accretion disk theory. The effect seems to depend on the black hole mass. While massive black holes $\left(M \gtrsim 10^{9} M_{\odot}\right)$ have nearly standard disks (but possibly larger in size, see Morgan et al. (2010), smaller black hole masses conform less with the standard model. For the latter, "disk" size is practically independent on wavelength for smaller-mass quasars (Blackburne et al. 2011).

Having similar luminosities $L \sim 10^{47} \mathrm{erg} \mathrm{s}^{-1}$, lower- and higher-mass bright lensed quasars have different Eddington luminosity limits. Moderately super-Eddington sources should form a scattering envelope that increases the apparent size of the object and makes the size dependence on wavelength weaker. In more detail we address this model in our recent paper Abolmasov \& Shakura (2012).

\section{Quasi-spherical envelope model}

Consider a spherically symmetric wind formed by a supercritical disk. It is reasonable to assume that at each radius $R$ the terminate velocity of the outflowing wind is virial: $v_{w}=\beta \sqrt{G M / R}$, where $\beta \sim 1$. The wind is effectively launched from the spherisation radius $R_{s p h} \simeq \frac{3}{2} \frac{G M}{c^{2}} \dot{m}$. Condition $\tau=1$ for the radial optical thickness leads to the following photosphere radius estimate:

$$
\frac{R_{1} c^{2}}{G M} \simeq \frac{f_{w}}{\sqrt{2}} \dot{m}^{3 / 2}
$$




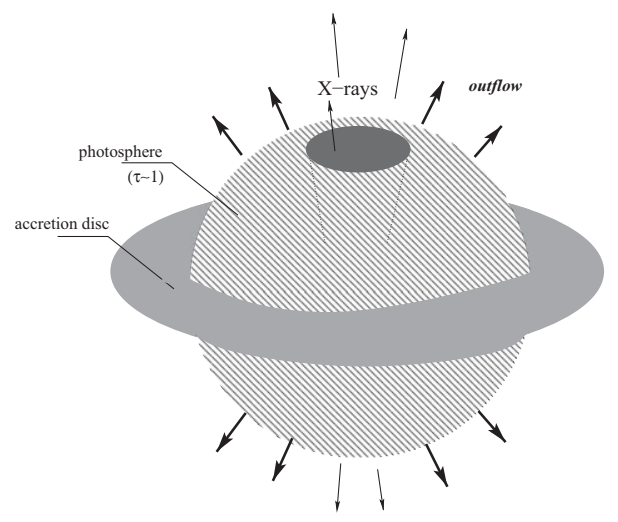

Figure 1. Qualitative sketch of the appearance of a super-Eddington quasar.

This may be both larger and smaller than the accretion disk radial scale: $R_{d} \simeq$ $10.2\left(\frac{\lambda_{e m}}{0.25 \mu}\right)^{4 / 3}\left(\frac{M}{10^{9} M_{\odot}}\right)^{-1 / 3} \dot{m}^{1 / 3} \times \frac{G M}{c^{2}}$. Note also that this photosphere is "grey" in the sense its size does not depend on the wavelength.

The issue of X-rays that are emitted by a considerably more compact region may be addressed in a more comprehensive model taking into account deviations from spherical symmetry. Both theory (Shakura \& Sunyaev 1973) and simulations (Ohsuga et al. 2005) predict that the wind formed by a supercritical accretion disk should have a conical optically thin funnel. The X-ray emission formed near the innermost stable orbit may reach the observer without scattering or after a single reflection from the funnel wall. In both cases, the size of the X-ray source will be considerably smaller than in the optical/UV range. The appearance of the quasar photosphere is illustrated by figure 1 .

\section{Summary}

We come to the conclusion that super-Eddington disk accretion may lead to formation of a scattering pseudo-photosphere capable of explaining the spatial properties of the emitting regions of distant lensed quasars in the optical/UV range. Our work was supported by the RFBR grant 12-02-00186-a.

\section{References}

Abolmasov, P. \& Shakura, N. I. 2012, ArXiv e-prints (arXiv:1208.1678)

Blackburne, J. A., Pooley, D., Rappaport, S., \& Schechter, P. L. 2011, ApJ, 729, 34

Morgan, C. W., Kochanek, C. S., Morgan, N. D., \& Falco, E. E. 2010, ApJ, 712, 1129

Ohsuga, K., Mori, M., Nakamoto, T., \& Mineshige, S. 2005, ApJ, 628, 368

Shakura, N. I., \& Sunyaev, R. A. 1973, A\&A, 24, 337

Wambsganss, J. 2006, in G. Meylan, P. Jetzer, P. North, P. Schneider, C. S. Kochanek, \& J. Wambsganss ed., Saas-Fee Advanced Course 33: Gravitational Lensing: Strong, Weak and Micro Part 4: Gravitational microlensing. pp. 453-540 